Communications in Physics, Vol. 28, No. 2 (2018), pp. 115-125

DOI:10.15625/0868-3166/28/2/12595

\title{
LOCALIZED SURFACE PLASMON RESONANCES WITH SPHERICAL METALLIC NANOPARTICLES
}

\author{
QUANG MINH NGO $a, b, \dagger$
}

${ }^{a}$ Institute of Materials Science, Vietnam Academy of Science and Technology, 18 Hoang Quoc Viet, Cau Giay, Hanoi, Vietnam

${ }^{b}$ Graduate University of Science and Technology, Vietnam Academy of Science and Technology, 18 Hoang Quoc Viet, Cau Giay, Hanoi, Vietnam

${ }^{\dagger} E$-mail: minhnq@ims.vast.ac.vn

Received 02 January 2018

Accepted for publication 19 April 2018

Published 16 June 2018

\begin{abstract}
In this work we review in part of our recent theoretical study on the electrical intensity enhancement in the dielectric medium surrounding metallic nanoparticles due to the effect of their localized surface plasmon resonance (LSPR). The known results in the case of a simple metallic spherical nanoparticle are presented and then extend them to the general case of complex network of the identical spherical metallic nanoparticles. For illustration, several typical lattices of identical spherical metallic nanoparticles will be treated. The findings of electrical intensity enhancements and plasmonic resonance wavelengths of the single and the network of the metallic nanoparticles are obtained based on the analytical expressions. The theoretical results were compared and shown the good agreement with simulation results. The simulation of the LSPRs and the electrical intensity enhancements was performed using the boundary element method.
\end{abstract}

Keywords: plasmonic, localized surface plasmon resonance (LSPR), dipole moment.

Classification numbers: 73.20.Mf; 78.67.Bf; 63.20.Pw; 41.20.Cv; 41.20.-q.

(C)2018 Vietnam Academy of Science and Technology 


\section{INTRODUCTION}

Localized surface plasmon resonance (LSPR) is known to be responsible for the electrical intensity enhancement, which determines the optical and spectroscopy properties and has led to advances in several areas of the science and technology such as nano-scale sensing and modern analytical techniques based on spectroscopy applications [1-9]. These resonances, associated with noble metallic nanostructures, create sharp spectral absorption and scattering peaks as well as strong near-field enhancement of the electromagnetics. Among these is the detection of molecules interactions near the particle surface through shifts in the LSPR spectral peak, namely surfaceenhanced Raman spectroscopy (SERS) [3-9]. The formation of inter-particle of noble metallic nanoparticles in the dielectric medium constitutes the basis for inter-particle plasmonic coupling responsible for SERS signal amplification, but understanding of its correlation with inter-particle spatial properties, particle sizes and the refractive indices of the surrounded media, remains elusive. Our previous work presented the basics of the theory of the light enhancement due to the LSPR effect in the metallic nanoparticles [10]. In this work we review in part of theoretical study on the electrical intensity enhancement in the dielectric medium surrounding metallic nanoparticles due to the effect of their LSPR. The simulations of using the boundary element method embedded in MNPBEM toolbox [11,12] are presented to confirm the findings of theoretical results and they have shown the good agreements. The achieved results of the electrical intensity enhancements for the single and coupled metallic nanoparticles embedded in dielectric medium in this work may provide a general guide line for plasmonic-based metallic nanoparticles design.

\section{THEORETICAL ANALYSIS}

For the description of the electromagnetic fields, the physical state of the electromagnetic fields are completely given by:

$$
\begin{gathered}
H(r, t)=\nabla \times A(r, t) \\
E(r, t)=\frac{i}{k \sqrt{\varepsilon_{m}}} \nabla \times H(r, t)
\end{gathered}
$$

where $\varepsilon_{m}$ is the dielectrical constant of the medium and $k=\sqrt{\varepsilon_{m}} \frac{\omega}{c}=\sqrt{\varepsilon_{m}} \frac{2 \pi}{\lambda}[13,14]$.

\section{II.1. Single metallic spherical nanoparticle}

Suppose that there exists a metallic spherical nanoparticle with radius $\rho$, and denote by $\varepsilon(\lambda)$ the effective dielectric constant or electric permittivity of the metallic material with respect to the electrical field in the monochromatic electromagnetic radiation with the wavelength $\lambda$, by $\varepsilon_{m}$ that of the dielectric medium surrounding the metallic nanoparticle. It was known that the electrical field:

$$
E^{0}(r, t)=e^{i(k r-\omega t)} E^{0}, k=\frac{2 \pi}{\lambda}
$$

of the linearly polarized monochromatic incident light induces following electrical dipole moment $p(R, t)$ inside the metallic spherical nanoparticle with the center located at some point $R$ in the space [14]:

$$
p(R, t)=4 \pi \varepsilon_{m} \rho^{3} L(\lambda) E^{0}(r, t)
$$


where

$$
L(\lambda)=\frac{\varepsilon(\lambda)-\varepsilon_{m}}{\varepsilon(\lambda)+2 \varepsilon_{m}}
$$

The induced electrical dipole moment located at some point $R$ emits into the surrounding space the electromagnetic radiation which will be called the induced electromagnetic field described by the vector potential $A_{\text {ind }}(r, t)$ or by a set of the induced electrical field $E_{\text {ind }}(r, t)$ and induced magnetic field $H_{\text {ind }}(r, t)$. It was shown that near the location of the induced dipole moment (4) the vector potential $A_{\text {ind }}(r, t)$ has the form

$$
A_{\text {ind }}(r, t)=-\frac{i k}{4 \pi \sqrt{\varepsilon_{m}}} \frac{e^{i k|r-R|}}{|r-R|} p(R, t)
$$

It follows that the induced electrical filed $E_{\text {ind }}(r, t)$ equals

$$
\begin{aligned}
E_{\text {ind }}(r, t)= & \frac{1}{4 \pi \varepsilon_{m}} e^{-i \omega t} e^{i k R} e^{i k|r-R|} \\
& \times\left\{[[n \Lambda p] \Lambda n] \frac{k^{2}}{|r-R|}+[3(n p) n-p]\left(\frac{1}{|r-R|^{3}}-\frac{i k}{|r-R|^{2}}\right)\right\}
\end{aligned}
$$

where

$$
n=\frac{r-R}{|r-R|}
$$

At a point $r$ very near to the metallic spherical nanoparticle, $k|r-R| \ll 1$, Eq. (7) has following approximate form

$$
E_{\text {ind }}(r, t)=e^{-i \omega t} e^{i k R} \frac{3(n p) n-p}{4 \pi \varepsilon_{m}} \frac{1}{|r-R|^{3}} .
$$

For the evident expression of the relationship between the induced electric field $E_{\text {ind }}(r, t)$ at a point $r$ outside the metallic spherical nanoparticle and the electric field $E^{(0)}(R, t)$ of the incident light at the point $R$ coinciding with the center of the metallic spherical nanoparticle we chose this center to be the origin of the coordinate system $(R=0)$ and the vector $r$ to be parallel to $E^{(0)}$. Then combining eqs. (4), (5), and (7), we have

$$
E_{\text {ind }}(r, t)=\chi(\lambda, d) E^{(0)}(0, t)
$$

where $d=r-\rho$.

$\chi(\lambda, d)$ versus to the wavelength $\lambda$ at different distances $d$ for the metallic spherical nanoparticle is the electric field enhancement, so that, $\chi^{2}(\lambda, d)$ is called the intensity enhancement.

\section{II.2. Network of identical metallic spherical nanoparticles}

Here we determine the enhanced electrical field in the spatial region surrounding a network of identical metallic spherical nanoparticles when this network is illuminated by a linearly polarized monochromatic light beam. Being induced by the electrical field of the incident light, each metallic nanoparticle as a dipole moment itself emits the electromagnetic radiation into the surrounding space. Therefore, the total electrical field at any point $r$ nearby but outside the metallic 
nanoparticles must be the superposition of the electrical emitted by the induced electrical dipole moments $p\left(R_{i}, t\right)$ and that of the incident light beam:

$$
E^{(t o t)}(r, t)=E^{(0)}(r, t)+\sum_{i} E_{\text {ind }}^{(i)}(r, t) .
$$

However, the total electrical filed $E^{(t o t)}\left(R_{i}, t\right)$ acting on the induced electrical dipole moment $p\left(R_{i}, t\right)$ located at the point $R_{i}$ is the superposition of the electrical field $E^{(0)}\left(R_{i}, t\right)$ of the

incident light and the electrical fields $E_{\text {ind }}^{(j)}\left(R_{i}, t\right)$ in the electromagnetic radiations emitted by other induced electrical dipole moments $p\left(R_{i}, t\right), j \neq i$ :

$$
E^{(t o t)}\left(R_{i}, t\right)=E^{(0)}\left(R_{i}, t\right)+\sum_{j \neq i} E_{\text {ind }}^{(i)}\left(R_{i}, t\right) .
$$

Because this total electrical field induces the electrical dipole moment $p\left(R_{i}, t\right)$, according to Eq. (4) we have

$$
p\left(R_{i}, t\right)=4 \pi \varepsilon_{m} \rho^{3} L(\lambda) E^{(t o t)}\left(R_{i}, t\right)
$$

Let us write the induced dipole moment Eq. (13) in the form

$$
p\left(R_{i}, t\right)=e^{i\left(k R_{i}-\omega t\right)} p^{(i)}
$$

From the Eq. (7) it follows that

$$
\begin{aligned}
E_{\text {ind }}^{(j)}\left(R_{i}, t\right)= & \frac{1}{4 \pi \varepsilon_{m}} e^{i\left(k R_{j}-\omega t\right)} e^{i k\left|R_{i}-R_{j}\right|} \\
& \times\left\{\begin{array}{l}
{\left[p^{(j)}-\left(n_{i j} p^{(j)}\right) n_{i j}\right] \frac{k^{2}}{\left|R_{i}-R_{j}\right|}} \\
+\left[p^{(j)}-3\left(n_{i j} p^{(j)}\right) n_{i j}\right] \times\left(\frac{i k}{\left|R_{i}-R_{j}\right|^{2}}-\frac{1}{\left|R_{i}-R_{j}\right|^{3}}\right)
\end{array}\right\}
\end{aligned}
$$

where

$$
n_{i j}=\frac{R_{j}-R_{i}}{\left|R_{j}-R_{i}\right|}
$$

\section{RESULTS AND DISCUSSION}

\section{III.1. Theoretical results}

Figure 1(a) shows the analytical result of intensity enhancement at the surface $(d=0)$ of single gold spherical nanoparticle surrounding by the titania medium for several nanopartilce radii, defined by the factor $\chi^{2}(\lambda, d)$ in Eq. (12). The dielectric constant of gold was experimentally measured by Johnson and Christy [15]. As can be seen, as the size of gold nanoparticle increases, the intensity enhancement increases, the resonant wavelength locates at $\sim 650 \mathrm{~nm}$. At plasmon resonance, strong electric intensity localizes at the surface of gold nanoparticle, for example, gold radius $\rho=10 \mathrm{~nm}$, at wavelength $\sim 650 \mathrm{~nm}$, the electric intensity is $\sim 1000$ times bigger than the incident intensity. Fig. 1(b) shows the intensity enhancement vs. distance $d$ for the radius $\rho=$ $10 \mathrm{~nm}$, and excitation wavelength $\lambda_{e x c}=650 \mathrm{~nm}$. The intensity enhancement decays quickly for increasing the distance $d$.

Consider first the simplest example case of a network of two identical gold spherical nanoparticles with the radius $\rho$ located at the distance $l(l \geq 2 \rho)$ as depicted in the inset of Fig. 2(a). This 
system is called a dimer. The intensity enhancements (normalized by the intensity enhancement of a single nanoparticle with same size) at the center of a dimer are defied by

$$
F_{\|}^{d}=\left(\frac{1}{1+\eta}\right)^{2}
$$

and

$$
F_{\perp}^{d}=\left(\frac{1}{1-\alpha}\right)^{2}
$$

for the incident polarizations of parallel and perpendicular to the inter-particle axis, respectively, with

$$
\eta=\frac{\rho^{3}}{l^{3}} L(\lambda) e^{i k l}\left(1-i k l-k^{2} l^{2}\right)
$$

and

$$
\alpha=2 \frac{\rho^{3}}{l^{3}} L(\lambda) \sum_{j \neq 0} \frac{e^{i k|j|}}{|j|^{3}}(1-i k l|j|)
$$
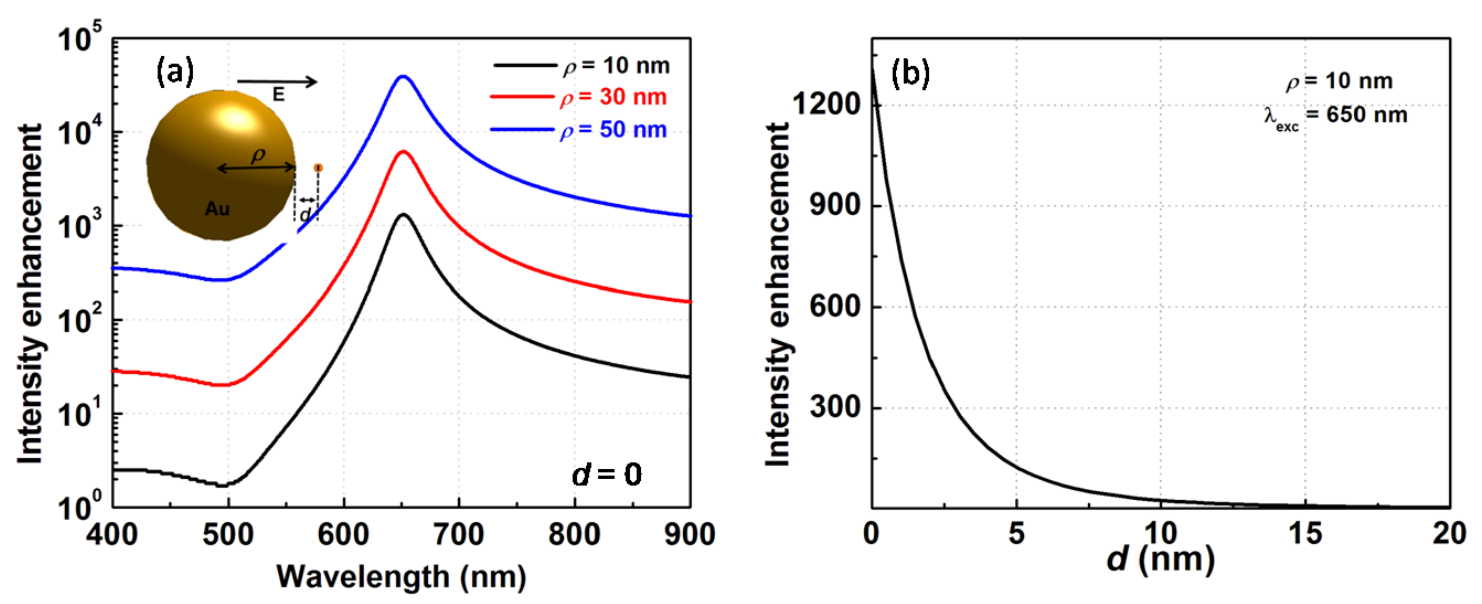

Fig. 1. (a) Intensity enhancement versus excitation wavelength at the surface $(d=0)$ for the spherical gold nanoparticles with different radii; (b) maximum intensity enhancement versus distance $d$ for the spherical nanoparticle with $\rho=10 \mathrm{~nm}$.

For the radius $\rho=10 \mathrm{~nm}$ and distance $l=25$, the normalized intensity enhancement of the dimer depends on the excitation wavelengths and plotted in Fig. 2(a) for both polarizations. The plasmon resonances are $\sim 695 \mathrm{~nm}$ and $\sim 625 \mathrm{~nm}$ for polarization of parallel and perpendicular, and at resonances, the normalized intensity enhancements are $\sim 12.5$ and 2.0 times, respectively. Fig. 2(b) shows the dependence of the maximum values of the normalized intensity enhancements for both polarizations on the distance between two centers of two gold spherical nanoparticles $d$, it decays quickly for increasing the distance $d$.

The second simple example of a network of identical metallic sphere nanoparticles is a linear chain depicted in the inset of Fig. 3(a). In the case of infinite linear chain and the polarized 
incident wave with the electrical field parallel to the direction of the chain, the normalized intensity enhancement at the center of the chain is given by:

$$
F_{\|}^{c}=\left(\frac{1}{1-\alpha}\right)^{2}
$$

where

$$
\alpha=2 \frac{\rho^{3}}{l^{3}} L(\lambda) \sum_{j \neq 0} \frac{e^{i k|j|}}{|j|^{3}}(1-i k l|j|) .
$$

and in the case of linearly polarized incident wave with the electrical field perpendicular to the direction of the chain, the normalized intensity enhancement is given by:

$$
F_{\perp}^{c}=\left(\frac{1}{1+\beta}\right)^{2}
$$

with

$$
\beta=\frac{\rho^{3}}{l^{3}} L(\lambda) \sum_{j \neq 0} e^{i k|j|}\left(\frac{1}{|j|^{3}}-\frac{i k l}{|j|^{2}}-\frac{k^{2} l^{2}}{|j|}\right) .
$$
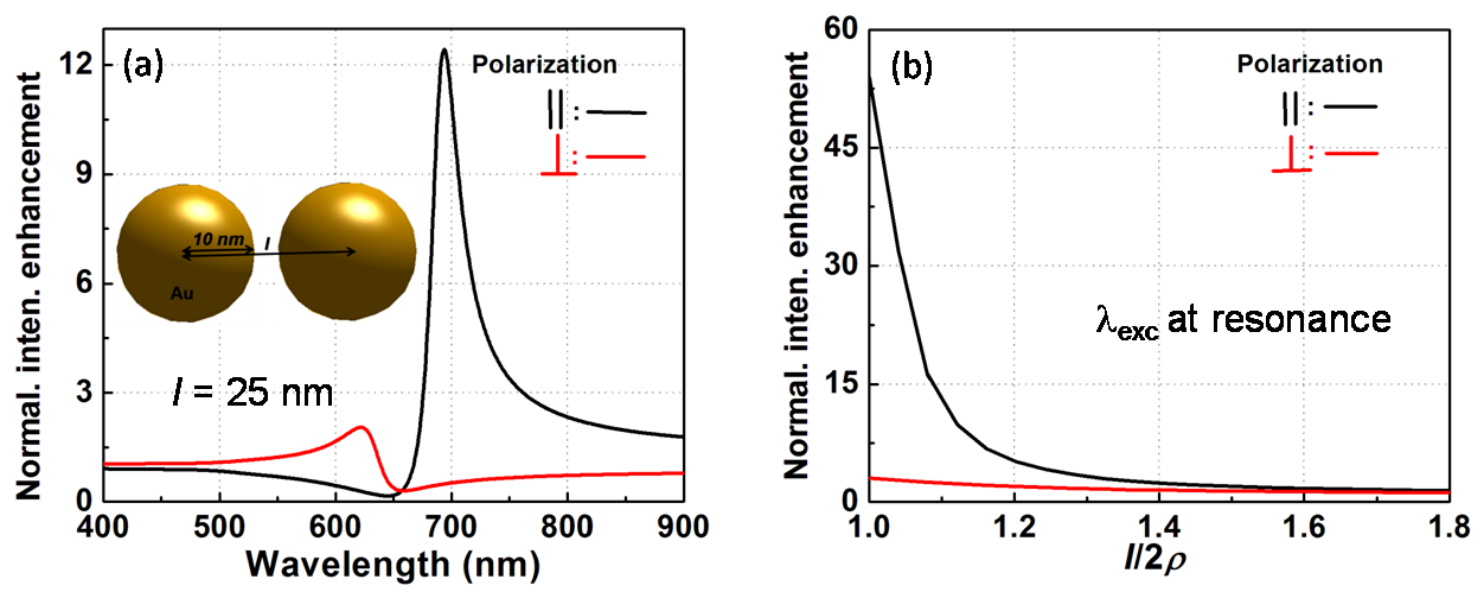

Fig. 2. (a) Intensity enhancement of the dimer with $\rho=10 \mathrm{~nm}, l=25 \mathrm{~nm}$ for both polarizations. (b) Dependence of the intensity enhancement of the dimer with $\rho=10 \mathrm{~nm}$ on the ratio $l / 2 \rho$.

According to Eqs. (21) and (23), the numerical calculations of intensity enhancements have been done for several numbers of identical gold spherical nanoparticles with the radius $\rho=10 \mathrm{~nm}$ and the distance of two nearest ones $l=25 \mathrm{~nm}$, the intensity enhancement is stable when $N=9$ as shown in Fig. 3(a).

The third network, which would be more often used, is that of a two-dimensional square lattice of identical gold spherical nanoparticles. Due to the translational invariance of the infinite 

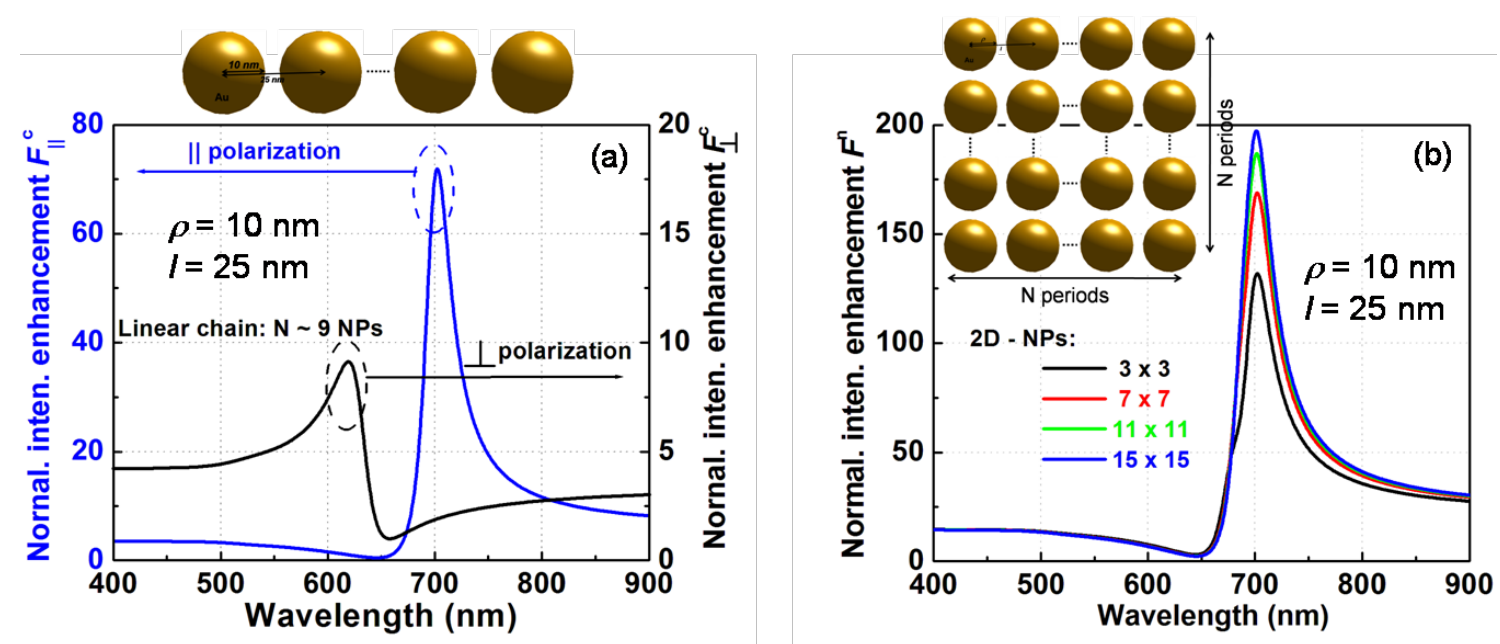

Fig. 3. Intensity enhancement of a chain (a) and the square networks (b) of gold spherical nanoparticles with radius $\rho=10 \mathrm{~nm}$ and the distance of two nearest ones $l=25 \mathrm{~nm}$.

square lattice, the solution for the network of infinite square lattice of identical gold spherical nanoparticles is given by:

$$
F^{n}=\left(\frac{1}{1-\varsigma}\right)^{2}
$$

where

$$
\begin{aligned}
\varsigma= & 2 \frac{\rho^{3}}{l^{3}} L(\lambda) \sum_{j \neq 0} \frac{e^{i k|j|}}{|j|^{3}}(1-i k l|j|)+2 \frac{\rho^{3}}{l^{3}} L(\lambda) \sum_{j^{\prime}>0} \sum_{j \neq 0} \frac{e^{i k \sqrt{j^{2}+j^{\prime 2}}}}{\left(j^{2}+j^{\prime 2}\right)^{3 / 2}} \\
& \times\left\{\frac{2 j^{2}-j^{\prime 2}}{j^{2}+j^{\prime 2}}\left(1-i k l \sqrt{j^{2}+j^{\prime 2}}\right)+k^{2} l^{2} j^{\prime}\right\},
\end{aligned}
$$

The intensity enhancement at the center of the square network of $N \mathrm{x} N$ gold spherical nanoparticles with $N=3,7,11$, and 15 are shown in Fig. 3(b). Other more complicated networks of identical metallic spherical nanoparticles can also be considered in a similar manner. From the expressions of the induced electrical dipole moments $p^{(i)}$ of the metallic spherical nanoparticles, it is now straightforward to calculate the total electrical field $E^{(t o t)}(r, t)$ of the resulting electromagnetic radiation in the space by using Eqs. (12)-(15).

\section{III.2. Simulation results}

The simulation of the surface plasmon resonance band and the intensity enhancement was performed using MNPBEM toolbox [11,12], which involved setting up the gold nanoparticles' boundary conditions and the titania medium surrounding the gold nanoparticles to calculate the optical scattering/absorption cross sections (SCS/ACS) and also the intensity enhancement patterns around the nanoparticles. Figures 4 and 5 show the simulation results for single and dimer of gold nanoparticles, respectively. The simulation results have good agreement with the theoretical 
(a)
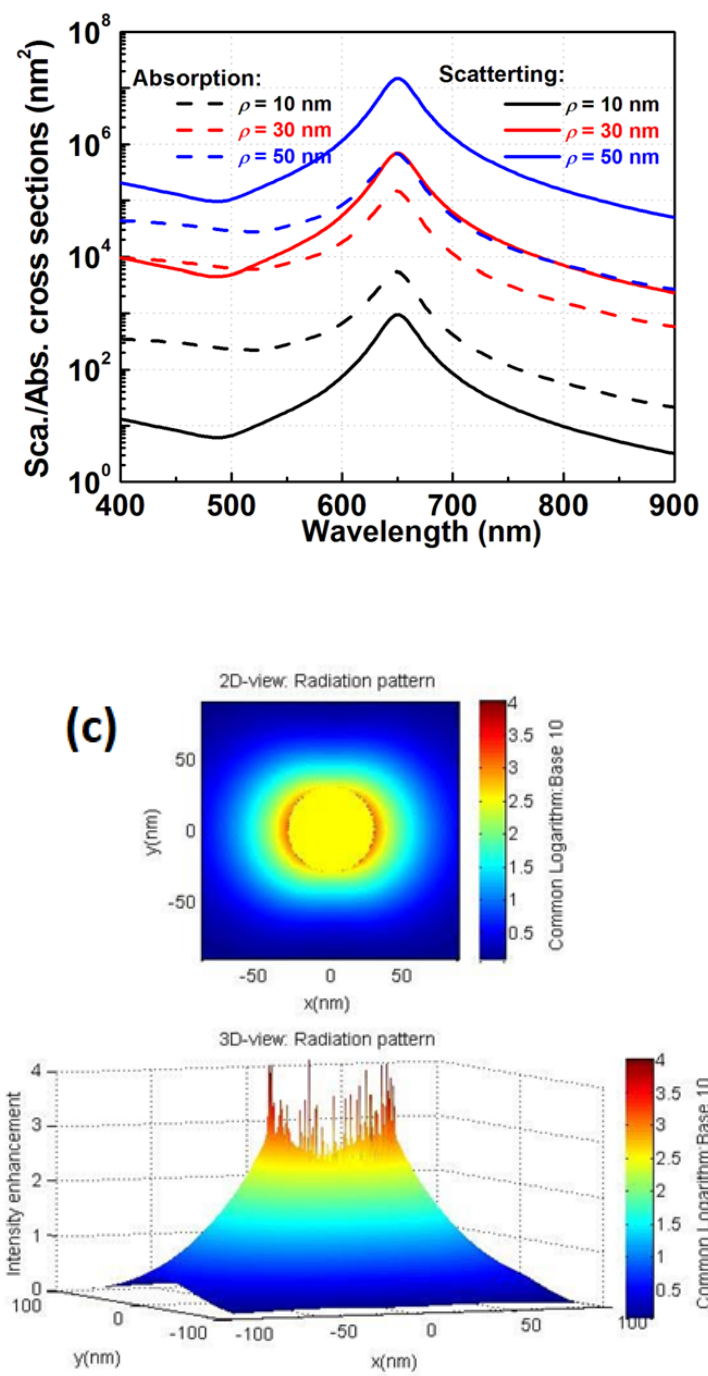

(b)
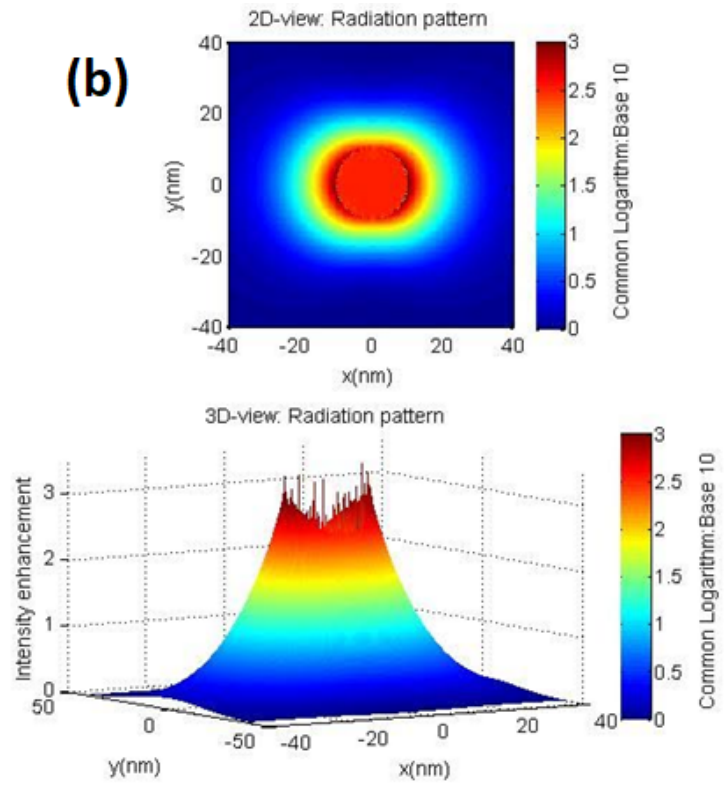

(d)
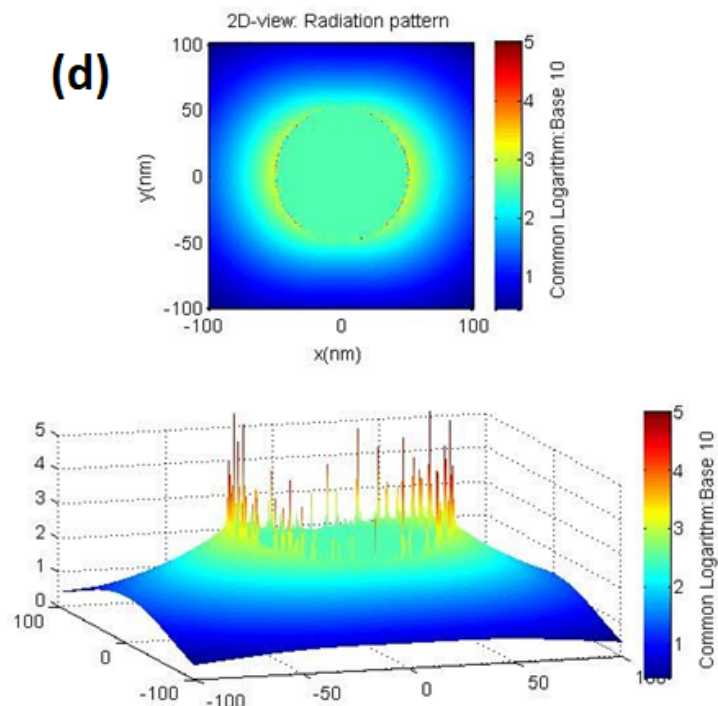

Fig. 4. (a) Scattering and absorption cross sections of the single gold nanoparticle immersed in the titania medium; (b), (c) and (d) 2D-views and 3D-views of intensity enhancement of single gold nanoparticle with radius of $10 \mathrm{~nm}, 30 \mathrm{~nm}$, and $50 \mathrm{~nm}$, respectively, under the excitation wavelength $\lambda_{\text {exc }}=650 \mathrm{~nm}$.

results as shown in Figs. 1 and 2 in resonant wavelengths and the electrical intensity enhancements. As seen in Fig. 4(a), the SCS/ACS peaks locate at around $650 \mathrm{~nm}$, which fits to theoretical results as shown in Fig. 1(a). Even the intensity enhancement and SCS units are different, but their tendencies on the radii of gold nanoparticles have shown the similar. That mean, increasing the 
radius of gold nanoparticle, the intensity enhancement and SCS increase. Figs. 4(b), 4(c), and 4(d) show the 2D-views and 3D-views of intensity enhancements of single gold nanoparticle under the excitation wavelength $\lambda_{\text {exc }}=650 \mathrm{~nm}$ for the radius of $10 \mathrm{~nm}, 30 \mathrm{~nm}$, and $50 \mathrm{~nm}$, respectively. As we can see, the intensity enhancements are $1.22 \times 10^{3}, 1.34 \times 10^{3}$, and $1.31 \times 10^{5}$, which show the same order of intensity enhancements as shown in Fig. 1(a).

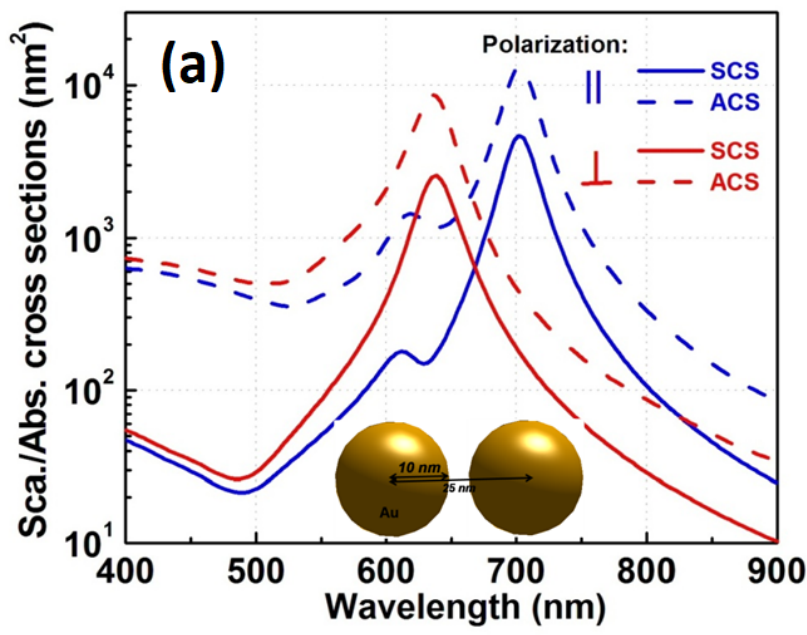

(b)

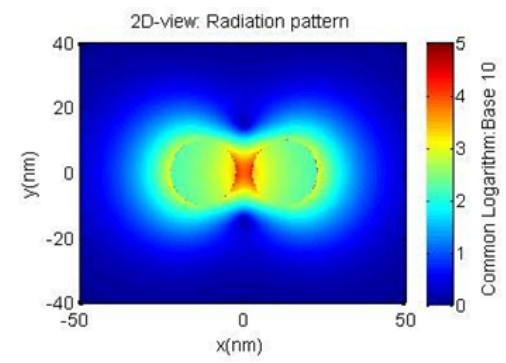

3D-view. Radiation pattern

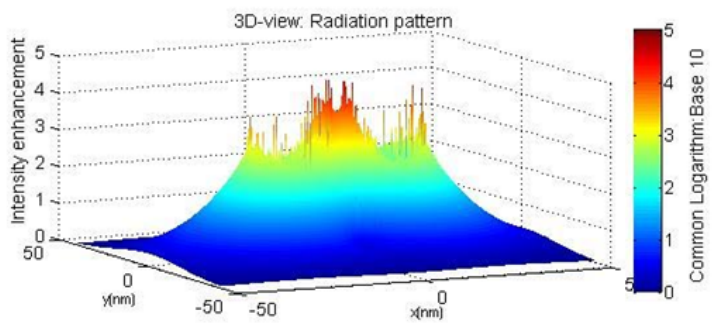

(c)

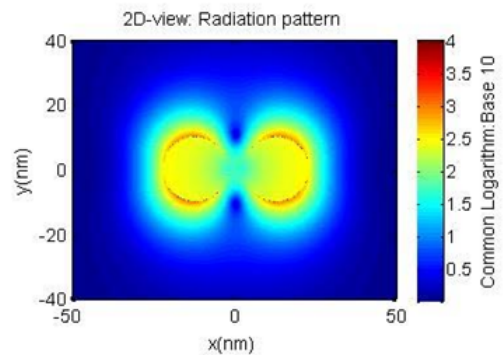

3D-view. Radiation pattern

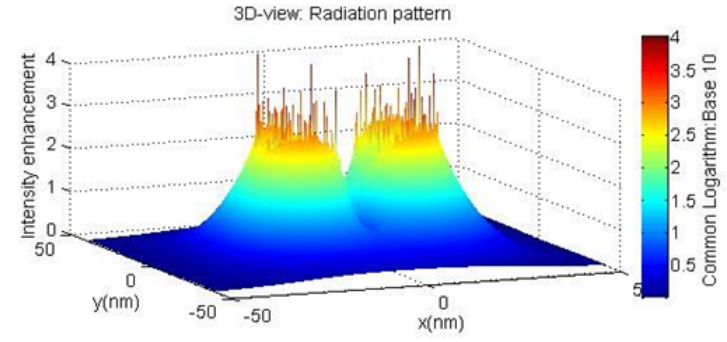

Fig. 5. (a) Scattering and absorption cross sections of the dimer of two identical gold nanoparticles immersed in the titania medium, with radius $\rho=10 \mathrm{~nm}$ and distance of two nearest ones $l=25 \mathrm{~nm}$; (b) and (c) 2D-views and 3D-views of intensity enhancement of dimer under the excitation wavelengths $\lambda_{e x c}=700 \mathrm{~nm}$ and $\lambda_{e x c}=630 \mathrm{~nm}$ for parallel and perpendicular polarizations, respectively. 
We also perform the simulation for the dimer of coupling two identical gold nanoparticles as shown in Fig. 5. Two excited polarizations of source of along and perpendicular to the interparticle axis, the radius of each gold nanoparticle of $10 \mathrm{~nm}$ and distance between them of $25 \mathrm{~nm}$ are used. As shown in Fig. 5(a), the resonant wavelengths of around $700 \mathrm{~nm}$ and $635 \mathrm{~nm}$ are achieved for polarizations of parallel and perpendicular to the inter-particle axis. There are a slightly shifts of resonant wavelengths between the theoretical and simulation results due to dielectric constant models. The 2D-views and 3D-views of intensity enhancements of the dimer for excited wavelength at resonances and polarizations of parallel and perpendicular are also simulated and shown in Figs. 5(b) and 5(c), respectively. As we seen, the "hot-spot" located at the center of the dimer when the excitation is along the inter-particle axis and two identical nanoparticles are close together. Two order intensity enhancement of the dimer compared to that of the single nanoparticle. In SERS applications, the hot-spots where the electromagnetic field is particularly intense, play a key role that provide molecular information through greatly enhanced Raman scattering from minute amounts of substance near nanostructured metallic surfaces. This work only provides a general numerical and simulation studies on the plasmonic enhancement by metallic nanoparticles in the dielectric medium. Other simulations of intensity enhancements in the cases of linear chain and network have been done but not shown here exhibit the same tendency as theoretical results.

\section{CONCLUSION}

In conclusion, the analytical expressions and algebraic equations determining the electromagnetic intensity in the dielectric medium surrounding these typical networks of metallic nanoparticles due to the effect of their localized plasmon resonance were presented theoretically and numerically investigated. From a review of the known results in the case of a simple metallic spherical nanoparticle, we extended these results to the general case of any network of many identical spherical metallic nanoparticles. The theoretical analysis was confirmed by a numerical study of the localized plasmon resonance and its plasmonic coupling using the boundary element method.

\section{ACKNOWLEDGEMENTS}

The author would like to express his gratitude to Academician Nguyen Van Hieu for suggesting this problem and for his encouragement. This research is funded by Vietnam National Foundation for Science and Technology Development (NAFOSTED) under grant number "103.032017.02”.

\section{REFERENCES}

[1] G. Mie, Ann. Phys. 25 (1908) 377.

[2] K. M. Mayer and J. H. Hafner, Chem. Rev. 111 (2011) 3828.

[3] Z. Liu, W. Hou, P. Pavaskar, M. Aykol, and S.B. Cronin, Nano Lett. 11 (2011) 1111.

[4] H. Wang, T. You, W. Shi, J. Li and L. Guo, J. Phys. Chem. C 116 (2012) 6490.

[5] Z. Skeete, H.-W. Cheng, J. Li, C. Salazar, W. Sun, Q.M. Ngo, L. Lin, J. Luo, and C.-J. Zhong, J. Phys. Chem. C 121 (29) (2017) 15767.

[6] Z. Skeete, H.W. Cheng, Q.M. Ngo, C. Salazar, W. Sun, J. Luo, and C.J. Zhong, Nanotechnol. 27 (2016) 325706.

[7] H. W. Cheng, S. I. Lim, W. Fang, H. Yan, Z. Skeete, Q.M. Ngo, J. Luo, and C. J. Zhong, J. Phys. Chem. C 119 (2015) 27786. 
[8] J. Li, Z. Skeete, S. Shan, S. Yan, K. Kurzatkowska, W. Zhao, Q.M. Ngo, J. Luo, and C.J. Zhong, Anal. Chem. 87 (2015) 10698.

[9] H.W. Cheng, Z. Skeete, Q. M. Ngo, J. Luo, and C. J. Zhong, J. Phys. Chem. C 17 (2015) 28529.

[10] B. H. Nguyen and Q. M. Ngo, Adv. Nat. Sci.: Nanosci. Nanotechnol. 5 (2014) 025010.

[11] U. Hohenester, Comput. Phys. Commun. 185 (2014) 1177.

[12] U. Hohenester and A. Trügler, Comput. Phys. Commun. 183 (2012) 370.

[13] S. A. Maier, Plasmonics: Fundamentals and Applications (New York: Springer, 2007).

[14] J. Jackson, Classical Electrodynamics (New York: Wiley, 1999) chapter 9.

[15] P. B. Johnson and R. W. Christy, Phys. Rev. B 6 (1972) 4370. 